\section{Indoor Positioning: Opportunities and Implementation Strategies of Bluetooth Low Energy}

Gl_Forum 2016, Vol.1

Page: 94-105

Full Paper

Corresponding Author:

christian.feil@stud.sbg.ac.at

DOI: 10.1553/giscience2016_01_s94

\author{
Christian Feil \\ RSA iSpace Salzburg, Austria
}

\begin{abstract}
We spend $90 \%$ of our time indoors. Buildings became larger and more complex (international airports, subway stations, shopping malls or hospitals for example). Our behaviour has changed. Given the possibilities of ubiquitous technology, we are used to finding out what our position is whenever we want - so far almost exclusively outdoors, however. We have little data on where and how people move indoors. There are various technologies for Indoor Positioning, but none of them dominates the market because of the very different requirements of the large range of applications. With Blvetooth Low Energy (BLE or Blvetooth Smart), a new technology has entered the market, and using it for Indoor Positioning is currently at the forefront of developments. Common positioning methods based on BLE include lateration, angulation, fingerprinting and proximity solutions. Most of them are based on the Received Signal Strength Indicator (RSSI). The RSSI correlates with the distance between transmitter and receiver antenna. However, the link is not as continuous as mathematical models suggest. The RSSI is influenced by many variables, some of which can be controlled and others not. This paper sums up general BLE positioning methods and the challenges they face with RSSI.
\end{abstract}

\title{
Keywords:
}

Indoor Positioning, Blvetooth Low Energy, Received Signal Strength Indicator, beacon

\section{Introduction}

Imagine this short story:

This is Kate, and Kate is in a panic. She is in a big subway station and feels as though she is being followed. After a while, she starts running, and scared as she is she loses her sense of direction. She runs into a dead end and behind her appears a robber asking aggressively for her money. What to do? She has no time to use her phone to call the police and no help seems to be at hand. She has no choice and hands over her handbag. Then she remembers and says "Please don't rob me". The robber laughs at her naïve words, takes the bag, and luckily leaves. Five minutes later, he is magically canght by the police while he is looking through the bag in another corner of the station. 
How did that happen? Kate has a Smart Device in her handbag, running a certain app in the background. The app has voice recognition and reacts silently to the code words used by Kate. From then on, it sends a message to the police every 30 seconds. The message contains information regarding her name, the emergency of a robbery, and the current position of the device. This does not sound too futuristic, does it? Everything is currently feasible - apart from the positioning, which is underground and GPS does not work there. This paper presents the possibilities of using the cheap and soon to be ubiquitous Bluetooth Low Energy beacon technology for getting a position inside buildings. Such clichéd crime scenarios aside, there are at least three facts that demonstrate the need to eradicate the remaining white spots, in indoor spaces.

a) We spend $90 \%$ of the time indoors (Worboys 2011). Of course, this percentage differs from person to person, depending on circumstances such as their employment, culture, preferences and so on. Nor does this figure say anything about the average person's activities, whether he/she is working, eating, sleeping or moving around. Nonetheless, it is remarkably high, and there is little or no data on where and how people move, once they have entered a building.

b) Buildings have changed, becoming larger and more complex. Think, for example, of international airports, subway stations, shopping malls or hospitals. Good architecture can counterbalance a lot of complexity, but this does not always work as expected if we think about our own experiences. There are strategies, ranging from signs and colour codes to building maps, that help orientation, but as yet there is no single and widely established positioning system.

c) Our behaviour has changed given the possibilities afforded by ubiquitous technology. In outdoor spaces, we are used to getting our position whenever we want to on mobile devices, be it on a car journey, in an unknown city, or when using all kinds of Location Based Services (LBS). As the high-accuracy positioning that is possible using mobile devices is mostly based on Global Navigation Satellite Systems (GNSS), it vanishes when a person enters a building and line of sight to the satellites is lost. Other positioning systems, like Wi-Fi or cellular radio systems, may work more or less indoors, but their accuracy is still very poor.

These three considerations might be reasons for the "why" of developing Indoor Positioning, and global players like Google, Apple and Microsoft have invested large sums of money to push it forward for their mass markets. The seamless integration of wellfunctioning positioning and navigation from outdoors to indoors has therefore become of key interest to the economy and science, as witness the ever-increasing number of publications addressing Indoor Positioning in recent years (as a query in the Scopus database reveals). The same query also revealed that very different domains show an interest in Indoor Positioning: the biggest sources for publications were in the engineering, computer science and social science sectors. This itself shows one of the main reasons why so far there is not just a single Indoor Positioning solution on the market: different requirements.

Outdoors, GNSS can cover most positioning requirements, as the factors of accuracy, time and costs are closely interconnected. For Indoor Positioning, there are many further factors, including coverage area, update rate, robustness, availability, scalability and number of users, 
to name but a few (Mautz, 2012). A one-size-fits-all technology is therefore very unrealistic for an indoor environment. An overview of the most common technologies currently in use is given in Table 1.

Table 1: Overview on Indoor Positioning technologies (adapted from Mautz, 2012)

\begin{tabular}{|l|l|l|l|}
\hline \multicolumn{2}{|c}{ Technology } & \multicolumn{1}{c}{$\begin{array}{c}\text { Typical } \\
\text { Accuracy }\end{array}$} & \multicolumn{1}{c|}{$\begin{array}{c}\text { Typical Coverage } \\
\text { in } \mathrm{m}\end{array}$} \\
\hline Cameras & $0,1 \mathrm{~mm}-\mathrm{dm}$ & $1-10$ & Application \\
\hline Infrared & $\mathrm{cm}-\mathrm{m}$ & $1-5$ & metrology, robot navigation \\
\hline Tactile \& Polar Systems & $\mu \mathrm{m}-\mathrm{mm}$ & $3-2000$ & people detection, tracking \\
\hline Sound & $\mathrm{cm}$ & $2-10$ & automotive, metrology \\
\hline Wi-Fi & $\mathrm{m}$ & $20-50$ & hospitals, tracking \\
\hline RFID & $\mathrm{dm}-\mathrm{m}$ & $1-50$ & pedestrian navigation,LBS \\
\hline Ultra-Wideband & $\mathrm{cm}-\mathrm{m}$ & $1-50$ & pedestrian navigation \\
\hline Highly Sensitive GNSS & $10 \mathrm{~m}$ & global & robotics, automation \\
\hline Pseudolites & $\mathrm{cm}-\mathrm{dm}$ & $10-1000$ & location-based-services \\
\hline Other Radio Frequencies & $\mathrm{m}$ & $10-1000$ & GNSS-challenged pit mines \\
\hline Inertial Navigation & 0,01 & $10-100$ & person tracking \\
\hline Magnetic Systems & $\mathrm{mm}-\mathrm{cm}$ & $1-20$ & pedestrian navigation \\
\hline Infrastructure Systems & $\mathrm{cm}-\mathrm{m}$ & building & hospitals, mines \\
\hline
\end{tabular}

In the form of Bluetooth Low Energy (BLE or Bluetooth Smart), a new technology entered the market around 2010. As the implementation of BLE for Indoor Positioning is currently at the forefront of development work, this paper is dedicated to analysing the potential of BLE for such a system. One of the core requirements of effective Indoor Positioning is the capability for real-time positioning. Many studies look at Indoor Positioning that works over Wi-Fi or similar systems, but few of them focus on the need for real-time positioning: people move constantly, and waiting at a certain spot for anything up to several minutes to get a position is not convenient for the user (Lymberopoulos et al., 2015).

\section{$2 \quad$ Methods \& Results}

In what follows, we will look more closely at BLE to provide an overview of its origins and legacy. Generally, the Received Signal Strength Indicator (RSSI) is used to reference BLE devices, but RSSI is a challenging variable as it is sensitive to external influences. These influences will be examined. The relevant positioning concepts will also be outlined and examined in the context of their potential for use with BLE.

\section{Bluetooth Low Energy}

BLE was integrated into Classic Bluetooth technology, as described in standard IEEE 802.15.1, firstly with version 4.0 in 2010. Research has been carried out on how to use Classic Bluetooth for Indoor Positioning, but these studies have identified Bluetooth Classic as rather challenging, because the technology bears a lot of constraints (Jung et al., 2007). 
Bluetooth Classic requires establishing connections between one device and up to 7 others to form a piconet. Several piconets can be connected to form so-called scatternets, but the connection procedure is cumbersome and slow, having a large overhead, and therefore does not allow real-time positioning (ibid.). For these reasons, Bluetooth Classic has never played a major role in Indoor Positioning.

With Bluetooth Smart, however, which uses an entire new Bluetooth stack, several of the drawbacks of Bluetooth Classic are eluded, as connectivity is no longer necessary, and security and privacy issues can be avoided simultaneously. BLE's current breakthrough on the market is thanks to beacon technology. A beacon is basically a small, generally batterydriven device that fires a signal regularly. In 2011, Apple introduced the iBeacon protocol, which defines the content of the signal of an according beacon in 31 bytes (Lindh, 2015). In 2014, Radius Networks released a similar open protocol, with AltBeacon; Google followed in 2015 with their protocols Eddystone-UID, Eddystone-URL and Eddystone-TLM, to integrate beacon technology in their Proximity Beacon API and Google Places API (Google, 2015). Today, there are dozens of beacon vendors (like Gimbal, easiBeacon, Ubudu or Estimote) that use one or other of these protocols. The substantial benefits of BLE using beacons are low cost (beacon prices currently start at $5 \$$ ), low energy (the battery can last for several years), and low maintenance (once configured and sited, no additional changes are necessary). Additionally, some of the beacons have sensors for temperature, light, acceleration or humidity and can be used to create small sensor networks. In terms of allowing Indoor Positioning to the broad mass of people, BLE has the important advantage of not requiring additional, dedicated devices: a common Smart Device supporting Bluetooth 4.0 or higher suffices.

With the advent of Bluetooth 4.1 in 2013 and 4.2 in 2014, BLE experienced several improvements and additions. A major consideration behind the updates was to make BLE fit for the Internet of Things. This is achieved through supporting Internet Protocol (IP) connectivity. In version 4.2, the Internet Protocol Support Profile (IPSP) is supposed to allow direct internet connectivity over IPv6/6LoWPAN. At the same time, frequencyhopping was improved to avoid interferences with LTE, and the data-exchange rate was also improved greatly. In addition, security issues were addressed. Of major importance in developing the capabilities of Indoor Positioning is the adding of simultaneous roles. A device with BLE 4.1 or higher can carry out different roles, like scanning and broadcasting, at the same time (Bluetooth SIG, 2013).

BLE 4.1 is just coming on to the market, and the first devices are becoming available. So far, however, beacon producers have not, in general, adapted to the new specifications.

\section{Referencing spatial systems with BLE}

In order to carry out positioning, several elements are necessary: first, the point of interest (POI) which is to be positioned; secondly, a reference system in an arbitrary number of dimensions; thirdly some way of producing output to give feedback on the relationship between the previous two. The connection from the reference system to the POI can be represented by a vector with the corresponding dimensions. The reference system must consist of at least one known point. For BLE, this means that both points need to be 
represented by Bluetooth Smart Devices, of which one must be the transmitter and one the receiver. In theory, both roles can be chosen freely, but in the implementation under realistic conditions there are differences. For this paper, let us assume that the known point is always represented by a BLE beacon and the POI is a common Smart Device.

The connection between beacon and Smart Device is achieved through the signal transmission. BLE is broadcast in the free, worldwide ISM (industrial, scientific and medical) band, at around $2.4 \mathrm{GHz}$. There are now two ways to measure the references between transmitting and receiving antennas:

a) Obtaining the distance by measuring the Time of Flight (ToF) of the signal: Like all radio frequency signals, the signal travels at the speed of light. The time difference between broadcasting and reception is measured, giving the ToF. This is the basic principle of GNSS systems, but whereas in GNSS systems the distances are long, in an indoor environment the ToF shrinks to mere nanoseconds, as the distances are short. This would need extremely precise and synchronized clocks, but the currently low prices of beacons and Smart Devices exclude this technology. An advanced option is therefore to use a Roundtrip ToF. Here, a mobile device such as a Smartphone transmits a signal that is answered immediately by the beacon. The distance is therefore covered in both directions, and synchronization of the clocks is not necessary. A system based on this could be successfully established for Wi-Fi (Ramírez, 2011). For BLE, this is still some way in the future. However, at the end of 2015, Bluevision, one of the big players in BLE and beacon technology, submitted an application for a patent for BLE beacons that use a Roundtrip ToF. Even though it might take several years for this to appear on the market, Bluevision proclaimed a breakthrough based on this technology (Sukhu, 2015).

b) Using the Received Signal Strength Indicator (RSSI): Each radio signal is broadcast with a certain amount of power, given as transmission power Tx. At the other end, the receiver can read the value of the received signal's power. Knowing both values, conclusions on the path loss can be drawn. According to Frii's free space propagation model, path loss for radio signals increases with distance. The relationship between the RSSI and distance is shown in the log-normal shadowing formula (Botta \& Simek 2013):

$$
d=d_{0} * 10^{\frac{R S S I_{0}-R S S I}{10 n}} \text { in }[\mathrm{m}]
$$

where $\mathrm{d}$ is the distance, RSSI0 is the RSSI measured at $\mathrm{d} 0$ (usually $1 \mathrm{~m}$ ), and $\mathrm{n}$ is the path loss parameter for external environmental distortion. The RSSI is based on a logarithmic scale and measured in decibel-milliwatts $(\mathrm{dBm})$. A rule of thumb is that for every $6 \mathrm{dBm}$, the distance doubles (see Figure 1). 


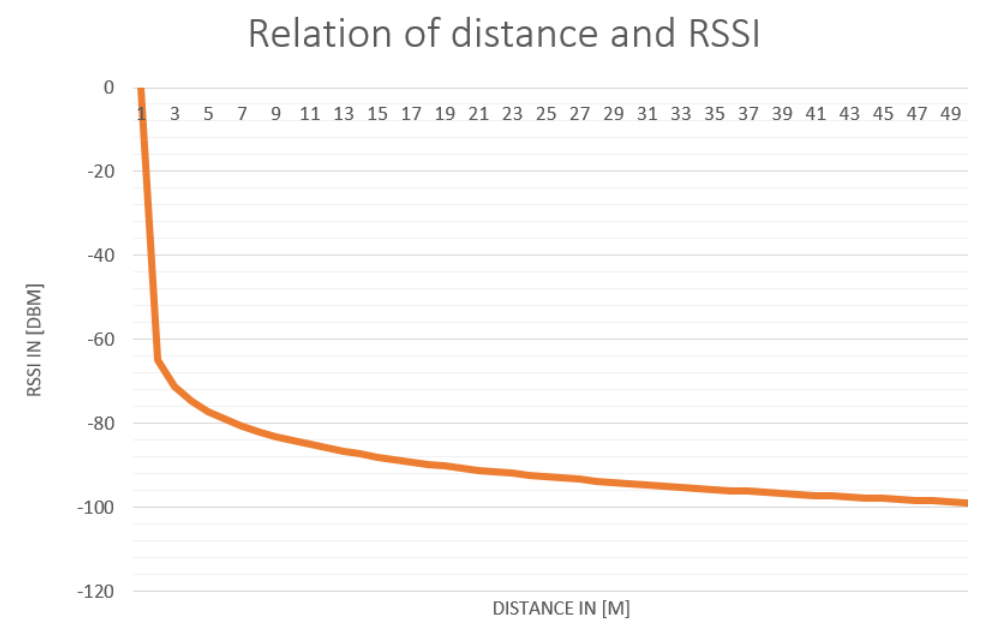

Figure 1: Ideal curve between RSSI and distance (adapted from Botta \& Simek, 2013)

So far, the ToF works only theoretically for BLE. Therefore almost all research to date uses the RSSI as reference.

\section{RSSI Measurements}

Under real conditions, the relation between the RSSI and the distance is never as clear as in the ideal mathematical models. Obstacles, multipathing from reflections, refractions and other influences distort the relation heavily. While these effects are hard to represent in detail and depend on the particular environment, the construction and handling of the BLE devices are also crucial.

In this ongoing research, some potential distortion factors were examined and the results are represented here. For all cases in the investigation, unless otherwise stated an easiBeaconPro and a Nexus 5 were used. The devices were placed 1 metre apart, maintaining the same orientation, and external influences were excluded as far as possible. All tests were performed multiple times, each one over several minutes, to gain statistical validity. Variables of interest were the mean RSSI, the standard deviation of the RSSI, and patterns in the RSSI raw data curve. For the purposes of the testing, a small android app was written. In order to determine whether a particular scenario had an influence on the RSSI, its statistical output was compared with the one from the initial setup. For example, the influence of Wi-Fi was examined by comparing the values for when Wi-Fi was shut down and for when it was running.

Initially, we tested whether changing the beacon's transmission frequency or transmission strength had any influence on the RSSI. The influence of the signal's power on the mean RSSI is obvious, as they are directly interconnected. More interesting was the potential effect on the RSSI variation. When these parameters were changed, a slight effect could be noticed. Also the transmission frequency had an impact on the RSSI variation; it therefore makes a difference whether a beacon is broadcasting in an interval of $100 \mathrm{~ms}$ or $1 \mathrm{~s}$. However, the 
correlation was not linear, and since the influence was weak, it can be disregarded in favour of stronger factors.

In another series of tests, the influence of other radio signals, for example from nearby Wi-Fi and BLE beacons, was measured. As Wi-Fi can use a similar frequency, of around $2.4 \mathrm{GHz}$, the literature warns about interferences (Lindh, 2015). According to our test results, however, the effect is hugely overestimated. No influence on the mean RSSI could be detected, and only a slight one on the standard deviation. But the tests did reveal that nearby beacons have a greater impact on the standard deviation. Here the variance of the RSSI is clearly larger, and it must be stated that the proximity of beacons distorted the signal strength.

In order to avoid interference with Wi-Fi, BLE broadcasts on three different channels. This is achieved by automated frequency-hopping, which means the beacons change channels regularly. This was associated with another phenomenon that we observed: when we looked at the results of the tests, the raw data of the RSSI measurements showed an explicit pattern, as shown in Figure 2.

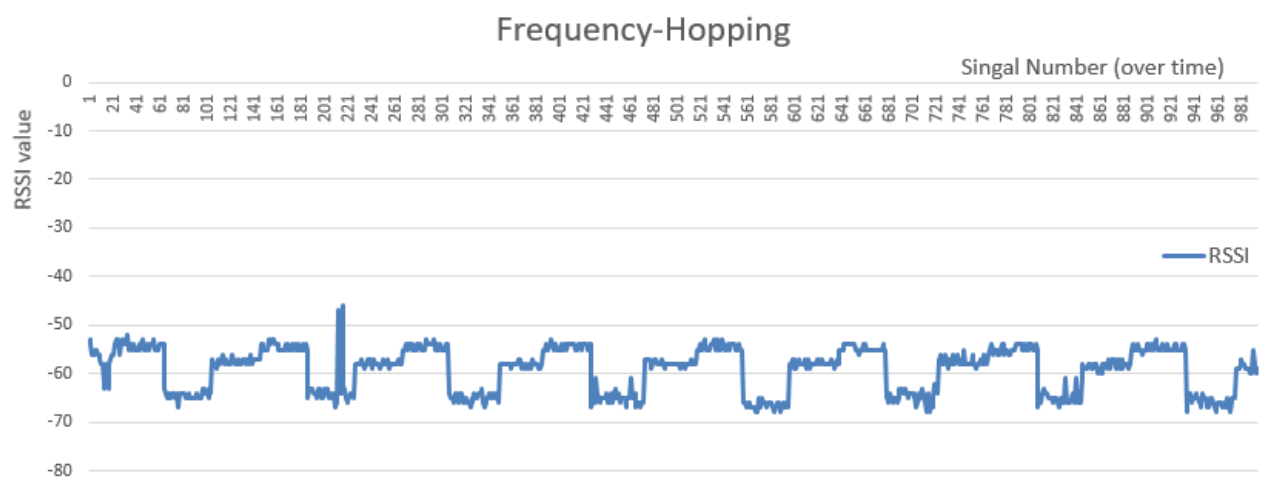

Figure 2: RSSI raw data with typical pattern

As seen in Figure 2, there are clearly three different RSSI levels that are repeated continuously during all measurements. Such a level is kept for around 5s. As the energy transmitted is connected with the frequency, it is very likely that the different RSSI levels correlate with the channels. Other research indicates that the channel has an influence not only on the overall energy but also on multipathing and the penetration behaviour of the signal, which are also related directly to the RSSI (Cinefra, 2013). It could not be verified whether this pattern came from frequency-hopping on the transmitter antenna or the receiving antenna scans on distinct channels for certain time frames.

Since the chipset quality and antenna sensitivity are of great importance, we tested for whether different beacon and phone models have any effect on the RSSI. For this, four different beacon models were tested. The differences both on the mean and standard deviation of the RSSI were noticeable but low. This might be explained by the fact that there are only a few chipset-producers for BLE beacons, but the vendors are numerous. A much 
more significant effect appeared when we compared the different phone models. The choice of the phone model had considerable influence on both the mean RSSI and the standard deviation of it. For some models, the mean RSSI was more than $10 \mathrm{dBm}$ lower than for others, and the standard deviation three times larger. Some devices also missed half of the signals broadcast, while others caught $90-100 \%$.

What the device model had already indicated was tested for again by altering the orientation of the antenna. Both the beacon and the phone were positioned at certain angles. It turned out that the antenna orientation has a very strong influence on the RSSI. Both the beacon orientation and the phone orientation have very high and nonlinear links to the RSSI variables. One explanation might be the modular construction of both, since thick metal pieces or the battery might block the signal at certain angles. Furthermore, the antenna is neither continuously omni-directional nor unidirectional. It is to be expected that different phone and beacon models react here very distinctively, since the structure and quality of the different phone parts vary for each model.

The effect of distance was examined. As, according to the log-normal shadowing model described in 2.2, the distance and RSSI are directly related, the mean RSSI behaved as expected with increasing distance. The interesting factor was therefore the standard deviation. With increasing distance, slightly more variance in the RSSI appeared, but the effect was weak.

Obstacles and interruption of line of sight lead to diminishing RSSI values (Kudak, 2014). This behaviour was also tested and could be verified. Also challenging is the fact that the human body, consisting to a large extent of water, absorbs BLE signals significantly, and people moving around therefore have a considerable impact on the RSSI.

Table 2 summarizes which changes in the setup led to noticeable influences on the RSSI. In order to make the table easily readable and to help with "what to look out for" in decisionmaking, the influences are stated using an ordinal scale:

Table 2: Influence factors on the RSSI

\begin{tabular}{|l|l|l|}
\hline Case (changes made regarding:) & Influence on mean RSSI & $\begin{array}{l}\text { Influence on SD of } \\
\text { RSSI }\end{array}$ \\
\hline Signal power & high & low \\
\hline Signal frequency & none & very low \\
\hline Wi-Fi nearby & none & low \\
\hline Beacons nearby & none & medium \\
\hline Beacon model & low & low \\
\hline Phone model & very high & very high \\
\hline Frequency-hopping pattern & very high & very high \\
\hline Antenna orientation & very high & very high \\
\hline Distance & high & low \\
\hline Obstacles & medium & medium \\
\hline & &
\end{tabular}


To summarize, there are influence factors that can be controlled to some extent and others that cannot. The orientation of the antenna in particular remains challenging, as it has a great influence but allows little control. The phenomenon supposedly connected to frequencyhopping is also difficult to manage, but this might be resolved in future hardware or software generations. Furthermore, the RSSI is a variable that produces a lot of noise and needs extensive filtering if valuable information is to be derived. Without filtering, the RSSI contains noise of around $10 \mathrm{dBm}$. As $6 \mathrm{dBm}$ means roughly double the distance between antennas (see Figure 1), it can be seen that RSSI noise is highly challenging.

\section{Discussion}

In this section we discuss the most common positioning techniques in relation to the difficulties identified above. There are other positioning methods, such as Neuronal Networks or Particle Filtering, but these so far have played a much less significant role. The most-used concepts are based on lateration, angulation, fingerprinting and proximity (Mautz, 2012). However, their suitability for use with RSSI-based calculations varies greatly.

Lateration (also tri- or multilateration) is using the distances between several known points and the POI. The basic principle is known from GNSS, where at least 4 satellites with known locations are necessary to determine a position on earth. The same procedure can be used indoors with BLE beacons, when the distance is calculated over ToF or by using the log-normal shadowing model of the RSSI. In 2D, 3 points with known coordinates are necessary; in 3D, 4 are required. Since most distances are not calculated exactly, the position in reality is only an estimation with changing accuracy. Especially for RSSI-based distances, this becomes a severe challenge, since - as explained in section 2 - the RSSI is influenced by various factors and is highly unstable. Without precise filtering, the distances calculated may vary by a factor of $2-3$, which reduces the reliability of the calculated position significantly. One way to deal with this is to take the mean over several seconds. Depending on the transmitting frequency, this could take up to half a minute to gain reliable values. Then, however, the conflict arises between desired accuracy and (near) real-time update rate.

Another option is to use angulation. The incoming angle or AoA (angle of arrival) of the signal can be measured using dedicated hardware. The hardware usually has an array of several dozen antennas and can therefore estimate the AoA. Another method is to use a highly directional rotating antenna. Since the Bluetooth signal is distinct and not continuous, however, it is mostly the first method that is used. If the AoA at two or more distinct known places is obtained, then the point of intersection of the directional lines is the POI. Such a system has recently been adopted by Qumppa's Intelligent Locating Technology ${ }^{\mathrm{TM}}$ (http://quuppa.com/technology/). Cisco is following a similar strategy with Cisco Hyperlocation $^{\mathrm{TM}}$ (http://www.cisco.com). Although the method is supposedly accurate, because it is less dependent on the RSSI fluctuation, when the direct line of sight is lost due to the presence of objects or people in between, positioning becomes very unreliable (Gauer 2015); the hardware costs, furthermore, are very high. .

Fingerprinting is a method used with BLE, usually by means of the RSSI. In this method, the POI is not referenced directly with the beacons but with pre-measured points in the area. 
Each point has unique characteristics. Therefore fingerprinting consists of a training phase in which all RSSI values of beacons surrounding a particular point are collected and saved in a database, together with the coordinates of that point. Later, in the online phase, the RSSI values obtained for a location are compared with each point in the database, and the point with greatest similarity is chosen as the position. Theoretically, other characteristics besides the RSSI can be taken into account. Indoor-Atlas ${ }^{\mathrm{TM}}$ (https://www.indooratlas.com) attempts to do the same thing using magnetic field variables, for example. The similarity between the measurements and points in the database can be assessed by calculating the least square error or k-nearest neighbour, or by using sophisticated approaches like artificial neural networks, Bayesian inference, or support vector machine (SVM) (Farid, et al. 2013). Using the RSSI as the decisive parameter here will again result in the question of how stable it is. Stability can be increased through extensive filtering and determining the mean over a longer period of time.

Proximity is the simplest form of positioning with BLE. In this case, the position of the closest beacon is taken. The closest beacon can be identified either from the RSSI, or by using the simple logic of "in range" or "not in range". As the transmission power of a beacon can be reduced from $50-80$ metres to $0.1-0.5$ metres, this method can result in very stable and reliable positioning. If several beacons are in reach, the RSSI and other parameters decide on the closest beacon.

\section{Conclusion}

For Indoor Positioning, there is not just one technical solution, nor for Indoor Positioning with BLE is there just a single positioning concept. "Better" in this case means better fitting to the requirements, and the requirements might be quite diverse in different applications. Accuracy is just one criterion out of many and is not always the most important one. If the system needs to be fast and reliable without requiring much configuration and to adapt automatically to changes in the environment, then proximity is a valuable method. However, the good functioning of proximity depends on the number of beacons used and how they are placed. In an environment without external influences (see section 2.3) and if real-time is not a necessity, fingerprinting can be considered. When costs are a less important consideration and accuracy is important over a small area, angulation systems might be chosen. When real-time is not important because of a steady environment, lateration approaches may be considered. Studies suggest that in different environments different positioning concepts work successfully (Dahlgren \& Mahmood, 2014; Cinefra, 2013), so there is no general answer or recommendation that can be given. Nevertheless, this study has demonstrated that using BLE has the potential to be efficient for Indoor Positioning as long as what is expected of the method remains in line with the limits of the current state of technology.

The next step is how to integrate the position in a system. For Indoor Positioning, a reference system and not only the coordinates are of interest. Where data models are concerned, three currently dominate the market, IndoorGML, IFC/BIM and CityGML, each focusing on different facets and each having advantages and disadvantages. However, as 
Indoor Positioning is not supposed to happen in empty space, the data models are a start in moving from obtaining mere coordinates to GIS integration, which ultimately can lead to visualization or statistical analysis.

With regard to statistical analysis, it is also necessary to mention potential misuse. Like any other positioning technology, BLE brings with it a risk of surveillance. Combining (Indoor) Positioning with geofencing or other event-processing, it can also be used to control people, such as employees or family members, and even to support modern forms of slavery (Dobson \& Fisher, 2003). A further concern is that the ubiquitous use of positioning and navigation in the outdoor environment that society already knows from GPS may intrude into the buildings equipped with Indoor Positioning and affect our behaviour. It is therefore important to maintain ethical boundaries when using this technology, and sociologists and psychologists are strongly advised to be vigilant with regard to these procedures.

BLE is a technology on the rise, and even though the strongest influence might come from advertising, once its additional benefits are seen, BLE technology will become part of our daily surroundings. Smart Devices like wearables will also become increasingly common, and it is likely that they will be used in combination with BLE. Finally, it should be mentioned that mixing Indoor Positioning with Big Data multiplies the potentials of both and can greatly enhance the analysis of customer movement, production cycles and more.

\section{References}

Bluetooth SIG, 2013. Press Releases. Updated Bluetooth ${ }^{\circledR} 4.1$ Extends the Foundation of Bluetooth Technology for the Internet of Things. [Online] Available at: http://www.bluetooth.com/Pages/Press-Releases-Detail.aspx?ItemID=197 [Accessed 2211 2015].

Botta, M. \& Simek, M., 2013. Adaptive Distance Estimation Based on RSSI in 802.15.4 Network. Radioengineering, 22(4).

Cinefra, N., 2013. An adaptive Indoor Positioning system based on Bluetooth Low Energy RSSI. Milano: Politecnico di Milano.

Dahlgren, E. \& Mahmood, H., 2014. Evaluation of Indoor Positioning based on Bluetooth Smart technology. Göteborg, Sweden: Chalmers University of Technology.

Dobson, J. E. \& Fisher, P. F., 2003. Geoslavery. IEEE Technology and Society Magazine, Volume 22(1), pp. 47-52.

Farid, Z., Nordin, R. \& Ismail, M., 2013. Recent Advances in Wireless Indoor Localization Techniques and System. Journal of Computer Networks and Communications.

Gauer, C., 2015. Delivering Location Based Services with Cisco Enterprise Mobility Services Platform. [Online]

Available at: http://www.cisco.com/web/offer/emear/38586/images/Presentations/P7.pdf [Accessed 1512 2015].

Google, 2015. Proximity Beacon API. [Online]

Available at: https://developers.google.com/beacons/proximity/guides [Accessed 1001 2016].

Jung, S., Chang, A. \& Gerla, M., 2007. New Bluetooth Interconnection Methods: Overlaid Bluetooth Piconets (OBP) and Temporary Scatternets (TS). Comput. Commun., Volume 30/10, pp. 2258-2273. 
Kudak, A., 2014. Evaluation und Anwendung aktueller Entwicklungen im Bereich Bluetooth Low Energy am Beispiel von iBeacon. Heidelberg : Universität Heidelberg - Hochschule Heilbronn. Lindh, J., 2015. Bluetooth ${ }^{\circledR}$ Low Energy Beacons. Application Report SWRA475, Dallas: Texas Instruments.

Lymberopoulos, D. et al., 2015. A Realistic Evaluation and Comparison of Indoor Location Technologies: Experiences and Lessons Learned. Seattle, Washington, ACM.

Mautz, R., 2012. Indoor Positioning Technologies. Zurich: ETH Zurich, Department of Civil, Environmental and Geomatic Engineering, Institute of Geodesy and Photogrammetry .

Ramírez, A., 2011. Time-of-flight in Wireless Networks as Information Source for Positioning. München: Universitätsbibliothek der TU München .

Sukhu, S., 2015. Bluvision achieves breakthrough with their ToF solution for indoor location using BLE technology. [Online]

Available at: http://bluvision.com/bluvision-achieves-breakthrough-tof-solution-indoor-locationusing-ble-technology/

[Accessed 2701 2016].

Worboys, M., 2011. Modeling indoor space. Chicago, Illinois, ACM, pp. 1-6. 